\title{
Comparative Studies on Lymphadenopathies in Canines and Bovines
}

\author{
Avantika Sharma* \\ Department of Veterinary Pathology, Guru Angad Dev Veterinary and Animal Sciences \\ University, Ludhiana, Punjab, India \\ *Corresponding author
}

\section{A B S T R A C T}

\begin{tabular}{l} 
Ke y w o r d s \\
$\begin{array}{l}\text { Bovines, Canines, } \\
\text { Cytology, } \\
\text { Lymph node, } \\
\text { Lymphadenopathies }\end{array}$ \\
\hline Article Info \\
\hline $\begin{array}{l}\text { Accepted: } \\
\text { 20 October } 2020 \\
\text { Available Online: } \\
10 \text { November } 2020\end{array}$ \\
\hline
\end{tabular}

Lymphadenopathy referred as lymph node enlargement is the most common problem in dogs, may be due to inflammatory or non-inflammatory conditions including neoplasm.In case of cattle and buffalo, Theileriosis showed lymph node enlargement as the most common complaint. It is not a specific disease entity but an important clinical finding; the cause should be ascertained to attempt treatment and prognosis. Enlargement of lymph nodes often occurs following the proliferation of resident normal cells or infiltration of outside normal or abnormal cells. Rarely, this enlargement can be caused by vascular changes (congestion, vascularization, and edema). Common causes of lymphadenopathy include neoplasia (lymphosarcoma and metastatic neoplasia), hyperplasia or reactive lymphadenopathy, lymphadenitis (neutrophilic, purulent, eosinophilic, pyogranulomatous, or granulomatous), immune stimulation, and extra medullary hematopoiesis. Lymph node cytology is an excellent way to evaluate a lymphadenopathy whether it is a single node enlarged, multiple nodes enlarged, or a generalized lymphadenopathy. This review, therefore, provides an overview of the various conditions that are associated with lymphadenopathies in canines and bovines.

\section{Introduction}

Lymphadenopathy is a commonly encountered condition in canines. It is not a specific disease entity but an important clinical finding; the cause should be ascertained to attempt treatment and prognosis (Thangapandiyan and Balachandran, 2010). Lymphadenopathy referred as lymph node enlargement is the most common problem in dogs, may be due to inflammatory or non-inflammatory conditions including neoplasm (Thangapandiyan and
Balachandran, 2014). Lymph node enlargement, referred to as lymphadenopathy, is a frequently encountered problem in veterinary patients. Evidence suggests that cytology serves as a very useful tool in the diagnosis of two common conditions viz., cutaneous/subcutaneous masses and lymphadenopathies in veterinary patients (Villiers and Dunn, 1998). Moreover cytological techniques have become an integral part of diagnosis in clinical cases of malignancy (Roszel, 1981). Being a simple and rapid procedure, the technique employs 
few resources to obtain the representative cells from most lesions. Although it is not possible to gain information on the tissue architecture, the cellular components, neoplastic and non-neoplastic cells could be interpreted clearly with the help of cytology (Morris and Dobson, 1992).

The following conditions are associated with lymphadenopathies in canines and bovines

\section{Canines}

Seasonal diseases such as ehrlichiosis and leishmaniasis should be considered a potential cause of the lymphadenopathy in regions where they are endemic, as well as in animals that have visited these areas (Gopegui et al., 2004). In dogs, frequent cause of lymphadenopathy was lymphoma (Day and Whitbread 1995). In one study infectious agent induced lymphadenopathies in dogs were studied. They reported T. evansi in the lymph node cytology smears indicating heavy trypanosomiasis. Lymphocytic cytoplasm showed Morula and elementary bodies of Ehrlichia species. Both extracellular and intra neutrophilicgamonts of $H$. canis were also found in the lymph node cytology smears (Thangapandiyan and Balachandran, 2013). In dogs, cutaneous mast cell tumors in the inguinal regions frequently metastasized to abdominal lymph nodes (Allenman and Bain, 2000). Other lymphadenopathies include cystic masses, mineral-associated lymphadenopathy (Day and others 1996), systemic histiocytosis, plexiform vascularisation (Welsh and others 1999), haemangioma and lymphangioma (Hogenesch and Hahn 1998).

\section{Lymphoma}

Neoplasia of the lymphoid system is one of the most common malignancies of domestic animals (Jarret et al., 1966). Canine lymphoma has often been proposed as an animal model of its human counterpart, the non-Hodgkin's lymphomas (NHL), for it is a spontaneous tumour in a non-inbred species that shares the environment of man (Strandstrom and Bowen 1982). Study on dogs suffering from lymphoblastic leukemia showed generalized peripheral lymphadenopathy and pyrexia. Here, diagnosis of lymphoma was made by cytology and tissue core biopsy (Thangapandiyan et al., 2013). Day and Whitbread 1995 reported that the mean age of dogs with lymphoma was $7.5+2.8$ years. Mild anaemia observed in this study was a common finding as also reported by (Teske1994). Hypercalcemia and high BUN values observed in this study in lymphoma were best documented by Weller et al1982.Gopeguiet al (2004) observed that lymph node enlargement is a common clinical sign in canine and feline patients which may reflect a variety of underlying disorders. In such cases it is necessary to distinguish between lymphoma or metastatic neoplasia, and other causes of lymphadenopathy, such as reactive hyperplasia or lymphadenitis, in order to institute appropriate therapy and establish a prognosis. They were of the opinion that seasonal diseases such as ehrlichiosis and leishmaniasis should be considered a potential cause of the lymphadenopathy in regions. In addition, they also suggested that the extent of the lymphadenopathy (solitary, regional or generalised) and the presence of additional lesions or abnormalities should be recorded. Thangapandiyan et al., (2013) evaluated the rate of incidence, age of occurrence and serum biochemical changes in dogs with lymphoma. The results showed generalized peripheral lymphadenopathy and pyrexia. Diagnosis of lymphoma was made by cytology and tissue core biopsy. They reported lower incidence rate and the mean + 
SE age of dogs $(8.25+0.64)$. The affected dogs also had a severe anaemia. In addition, hypercalcemia and high BUN values were observed in lymphoma.

\section{Lymphosarcoma in dogs}

Weller et al, (1982) analysed twenty-four dogs with lymphosarcoma accompanied by hypercalcaemia and studied clinical, haematological and biochemical parameter. Male dogs were more frequently affected than female dogs. Anorexia, weight loss, muscular weakness, depression, polydipsia, polyuria, and bilateral peripheral lymphadenopathy were the most frequently observed clinical signs and physical findings. Hypercalcaemia, creatinaemia, azotaemia, hypercalciuria, hyposthenuria, and decreased endogenous creatinine clearance were the most frequent laboratory abnormalities. Detailed gross and histopathologic examinations revealed the multicentric anatomic form of lymphosarcoma, absence of skeletal metastases, and normal parathyroid glands. Histologic staging revealed five diffuse histiocytic lymphomas, and five diffuse lymphocytic poorly differentiated lymphomas.

\section{Haematopoietictumours those originating from the lymphatic tissues in dogs}

Teske et al, (1994) analysed that the haematopoietic tumours, in particular those originating from the lymphatic tissues, are among the most frequently occurring neoplasms in the dog. They proposed canine lymphoma as an animal model of its human counterpart, the non-Hodgkin's lymphomas (NHL) as it is a spontaneous tumour in a noninbred species that shares the environment of man. Thangapandiyan and Balachandran (2010) reported that lymphadenopathy is a commonly encountered condition in canines. They made cytological diagnosis from109 cases and observed 52 reactive hyperplasia, 25 neutrophilic lymphadenitis, 15 eosinophilic lymphadenitis, 12 metastatic lymphadenopathies, 4 lymphomas and 1 plasmacytoma. They concluded that the FNAB technique and Romanowsky's stains were found to be the easy and rapid methods for lymph node sampling and staining respectively.

\section{Thecytologic patterns in LNs of dogs with different phases of canine monocyticehrlichiosis (CME)}

Mylonakis et al., (2011) compared the cytologic patterns in LNs of dogs with different phases of canine monocyticehrlichiosis (CME), to investigate the association of cytologic pattern and presence of Ehrlichia spp. morulae, and compared the patterns of lymphoid reactivity between dogs with CME and those with non infectious causes of lymphoid hyperplasia. They reported that dogs with myelosuppressive CME have more lymphoid cytologic abnormalities than dogs with nonmyelosuppressive CME. They were of the opinion that $\mathrm{LN}$ plasmacytosis is the major pattern of lymphadenopathy in dogs with $\mathrm{CME}$ and was found more frequently in dogs with CME than in dogs with non-infectious causes of lymphadenopathy. de Castro et al., (2004) revealed pale mucous membranes, generalized lymphadenopathy, splenomegaly, edema and ascites in dogs having CME. Microcopically, the main lesions were: lymphoreticular hyperplasia in cortical areas of lymph nodes and spleenic white pulp, periportal accumulation of mononuclear cells and centrolobular fatty degeneration of the liver.

\section{Infectious agents Induced Lymphadenopathies in Dogs}

Thangapandiyan and balachandran (2013) studied the Infectious agents Induced 
Lymphadenopathies in Dogs. Lymphadenopathy is a commonly encountered problem in canine practice. They reported $T$. evansi in the lymph node cytology smears indicating heavy trypanosomiasis. Lymphocytic cytoplasm showed Morula and elementary bodies of Ehrlichia species. Both extracellular and intra neutrophilicgamonts of $H$. caniswere also found in the lymph node cytology smears in dogs having lymphadenopathies.

\section{Bovines}

\section{Lymph node enlargement in bovine Theileriosis}

In case of a cow which had generalized lymph node enlargement, cachexia, severe signs of anemia and decreased milk production. Analysis of the hemogram, revealed severe non regenerative anaemia, as evidenced by markedly decreased RBC count, PCV and haemoglobin concentration and no evidence of polychromasia and basophilic stippling. Severe leukopenia was also present. Romanowasky stained Imprints of the prescapular lymph nodes revealed trypomastigotes of Trypanosoma vivax among the RBCs and lymphocytes (Salgado et al., 2011). Theileriosis is a tick borne blood protozoan disease caused by various Theileria species affecting cattle and buffaloes (Brown 1990). The affected animals show fever, anemia, enlargement of lymph nodes, anorexia and progressive loss of body weight (Gill et al., 1977; Aulakh and Singla2006). Koch's blue bodies were observed in the proliferating lymphocytes as well as extra cellulary in the affected lymph nodes (Gupta et al, 2017). Chronic lymphatic leukemia was observed in cows, sheep and goats suffering from Enzootic bovine leukemia caused by bovine leukemia virus. It was characterized by long lasting lymphocytosis (Burny et al., 1985). Gill et al., (1977) observed the symptoms and pathology of experimental bovine tropical theileriosis (Theileria annulata infection). They reported enlargement of local lymph gland, fever, haemorrhages on visible mucous membranes. They also observed anaemia and jaundice particularly in subacute and chronic forms of the disease. Post-mortem findings reported were oedema, enlargement and haemorrhages in lymph glands. The microscopic lesions were characterized by hyperplasia of lymphoid cells at the haemopoietic centres in lymph glands and spleen followed by regression and degeneration of the germinal centres. Gupta et al., (2017) studied the Molecular and pathological studies on natural cases of bovine theileriosis. They conducted studies on 32 cases of bovine lymphadenopathies. They collected samples from affected lymph nodes which confirm Theileria annulata infection in five out of six cases. They concluded that pathological studies and cytokine analysis of lymph nodes are of paramount importance in disease diagnosis and associated immune response of the animal with lymphadenopathies.

\section{Lymphosarcoma in heifer}

Dalgleish et al., (1991) reported a typical case of lymphosarcoma in heifer. A clinical diagnosis of lymphosarcoma (sporadic bovine leucosis) was derived from a fine needle aspiration of a skin lesion. Post mortem and histopathological examination confirmed a multicentric lymphosarcoma with widespread infiltration into many tissues recogniosed as predilection sites for this type of tumour. In case of cutaneous lymphosarcoma in heifers, the mandibular, prescapular, prefemoral and supramammary lymph nodes were all enlarged (Marshak et al., 1966). Enlargement of lymph node was reported and the hematologic analysis revealed mild neutrophilic leukocytosis, and monocytosis compatible with a chronic inflammatory response (Dubreuil et al., 1988). 
Lymphadenopathy in a cow with severe anemia

Salgado et al., (2011) studied lymphadenopathy in a cow with severe anemia. They observed that the cow had generalized lymph node enlargement, cachexia, severe signs of anemia, and decreased milk production. Analysis of the hemogram revealed severe non regenerative anemia, as evidenced by markedly decreased RBC count and haemoglobin concentration and no evidence of polychromasia and basophilic stippling. Severe leukopenia was also present. The cow was euthanized, and a necropsy was performed. Severe pallor of mucous membranes, body wall, and musculature and moderate serous fat atrophy were noted. Imprints of the prescapular lymph nodes were prepared, and the slides were stained with a quick Romanowsky stain. They concluded that Trypanosoma species and other protozoan and rickettsial organisms, such as Babesia species and Anaplasma species were important as they cause diseases with similar clinical signs. Hajihassaniet al 2020 reported various species of Trypanosoma parasites to infect several wild and domestic animals worldwide. Physical examination revealed fever, enlarged subscapular lymph node, and pale mucosa, regenerative anemia, and progressive weight loss has been revealed in sporadic clinical cases in domestic animals.

\section{Diagnostic aspect for lymphadenopathies}

\section{Fine-needle aspiration (FNA) cytology}

Among the diagnostic tests, fine-needle aspiration (FNA) cytology is considered as an appropriate procedure in terms of its potential complications, time required, expense, sensitivity and specificity (Teske et al., 1994; Langenbach et al., 2001). Lymph node FNAC is a valuable tool in small animal practice, and a simple and safe procedure to diagnose the cause of lymphadenomegaly (Gopegui et al., 2004). As a result, FNA cytology has been considered a reliable method of evaluating metastasis in the regional lymph nodes in dogs and cats with solid tumours (Langenbach et al., 2001). Thangapandiyan and balachandran (2014) compared the cytological and histopathological techniques in the diagnosis of canine lymphadenopathies. A total of 29 Fine Needle Aspiration Biopsy (FNAB) samples and 21 tissue samples were collected from dogs with the complaint of lymphadenopathy. They observed $77.78 \%$ of carcinomas and $50 \%$ of sarcomas were identified on lymph node cytological smear and $57.14 \%$ of carcinomas on histological sections. The correlation found between the cytomorphological findings and histopathological features clearly substantiated the usefulness of cytology in the differential diagnosis of lymphadenopathies. They were of the opinion that FNAB technique was highly sensitive in detecting metastatic lymphadenopathies. Langenbach $e t$ al., (2001) determined Sensitivity and specificity of methods of assessing the regional lymph nodes for evidence of metastasis in dogs and cats with solid tumors. They suggested that fine-needle aspiration may be a sensitive and specific method of evaluating the regional lymph nodes in dogs and cats with solid tumors, because results correlated well with results of histologic examination of the entire lymph node. The tumors included 18 sarcomas, 16 carcinomas, 7 mast cell tumors, and 3 other tumors. Carcinomas were more likely to have metastasized to the regional lymph node than were sarcomas.

Air dried FNAB smears yield cells with optimal cytoplasmic and nuclear details (Morris and Dobson, 1992). The nuclear and nucleolar details were sufficient to differentiate neoplasia and inflammation and 
for cytological evidence of malignant potential with Romanowsky's stain (Meinkoth and Cowell, 2002). When air dried cells were stained with Haematoxylin and Eosin (H\&E) there was marked loss of nuclear details when compared with the cells that were rehydrated or immediately fixed with 95\% ethanol (Lumsden and Baker, 2000)

\section{MRI and AUS for detection of abdominal lymphadenopathy}

Anderson et al., (2015) compared the abdominal ultrasound and magnetic resonance imaging for detection of abdominal lymphadenopathy in dogs with metastatic apocrine gland adenocarcinoma of the anal sac. The data suggested that MRI is more sensitive than abdominal ultrasound (AUS) for detecting sacral abdominal lymphadenopathy in dogs with apocrine gland adenocarcinoma of the anal sac (AGAAS). (Stahle et al., 2019) diagnosed dogs with solid tumors, regional lymph node involvement or evidence of distant metastasis which can predict worse prognoses and significantly decreased survival. Lymph node size alone has been shown to be insufficient as a predictor for the accurate clinical staging of some canine neoplasia. They evaluated diffusion-weighted MRI and ADC as potential methods for detecting metastatic lymph nodes in dogs with naturally occurring disease.

\section{Radiographic visualization for the detection of lymphadenopathy}

Murphy et al., (2018) determined the efficacy of radiographs for the detection of sublumbar lymphadenopathy and identified radiographic changes that suggested nodal enlargement. They reported that radiographic visualisation of sublumbar lymph nodes was associated with marked enlargement (>17 mm), which should raise suspicion for metastatic infiltration. However, lack of visualisation does not exclude mild to moderate lymphadenopathy and further imaging is to be warranted.The thoracic and abdominal radiographs of dogs with multlcentric lymphoma were examined to identify the radiological abnormalities. The absence of radiological abnormalities proved as a positive prognostic indicator but, in general, radiology had no place as a prognostic Indicator for the Individual patient (Blackwood et al., 1997).

In conclusion the lymphadenopathy refers to the enlargement of lymph nodes. This is a frequent clinical problem. Infection, autoimmune disorders, and neoplasms can induce lymphadenopathy. Enlargement of lymph nodes is a common clinical finding with a wide range of underlying disease states. Lymphadenopathy may involve peripheral (palpable) lymph nodes and/or internal visceral lymph nodes. Lymphadenopathy may be localised (solitary or regional) or generalized in distribution. It might also encompass situations where lymph nodes are reduced in size, for example in senility, cachexia, primary immunodeficiency disease or with viral infection or immunosuppression that depletes lymphoid tissue. Further, lymph nodes might display pathological change (e.g., the presence of metastatic tumour) without necessarily being enlarged. Lymphnode FNA cytology is to diagnose the cause of lymphadenopathy.

\section{References}

Allenman, R. A., and Bain, P.J. 2000. Diagnosing neoplasia: The cytological criteria for malignancy. Vet. Med, 95:204-223.

Anderson, C.L., MacKay, C.S., Roberts, G.D., and Fidel, J. 2015. Comparison of abdominal ultrasound and magnetic resonance imaging for detection of abdominal lymphadenopathy in dogs 
with metastatic apocrine gland adenocarcinoma of the anal sac. Veterinary and comparative oncology, 13(2): 98-105.

Aulakh, G.S., and Singla, L.D. 2006. Clinicohaematobiochemical observations on bovines naturally infected with Theileria annulata. Journal of Veterinary Parasitology,20:49-52.

Blackwood, L., Sullivan, M., and Lawson, H. 1997. Radiographic abnormalities in canine multicentric lymphoma: a review of 84 cases. Journal of small animal practice, 38(2): 62-69.

Brown, C.G.D. 1990. Control of tropical theileriosis (Theileria annulata infection) of cattle. Parassitologia, $32: 23-31$.

Burny, A., Bruck, C., Cleuter, Y., Couez, D., Deschamps, J., Ghysdael,J., et al., 1985. Bovine leukemia virus, a versatile agent with various pathogenic effects in various animal species. Cancer Research, 45(9): 4578-4582.

Dalgleish, R., Callanan, J.J., and McNeil, P.E.1991. An atypical case of lymphosarcoma (sporadic bovine leukosis) in a heifer. Veterinary Record, 129: 308-310.

Day, M.J., and T.J Whitbread. 1995. Pathological diagnoses in dogs with lymph node enlargement. Veterinary Record, 136: 72-73.

Day, M J., Pearson, G R., I Ucke, V.M., Ilane, S.J and Sparks, R.S.J. 1996. Lesions associated with mineral deposition in the lymph node and lung of the dog. Veterinary Pathology, 33: 29-42

de Castro, M.B., Machado, R.Z, de Aquino, L. P. C. T., Alessi, A. C and Costa, M.T. 2004. Experimental acute canine monocytic ehrlichiosis: clinicpathological and immunopathological findings. Veterinary parasitology, 119(1): 73-86.

deGopegui, R. R., Peñialba, B., and Espada, Y. 2004. Causes of lymphadenopathy in the dog and cat. Veterinary Record, 155(1): 23-24.
Dubreuil, P., Lanevschi, A., Perrone, M.A. and Desnoyers, M. 1998. Atypical sporadic lymphosarcoma in a 7-month-old Holstein heifer. The Canadian Veterinary Journal, 39(7): 431.

Gill, B.S., Bhattacharyulu, Y., andKaur, D. 1977. Symptoms and pathology of experimental bovine tropical theileriosis (Theileria annulata infection). Annales de Parasitologie Humaine et Comparee, 52:597-608.

Gupta, A., Gupta, K., Leishangthem, G.D., Bal, M.S., Sood, N.K., and Singh, A. 2017. Molecular and pathological studies on natural cases of bovine theileriosis. Journal of Parasitic Diseases, 41(1): 211-218.

Hajihassani, A., Maroufi, S., Esmaeilnejad, B., Khorram, H., Tavassoli, M., DalirNaghadeh, B., et al., 2020. Hemolytic anemia associated with Trypanosoma theileri in a cow from Kurdistan province, West of Iran. In Veterinary Research Forum. 11( 2); p. 191.

Hogenesch, H., and Hahn, E.E. 1998. Primarily vascular neoplasms of lymph nodes in the dog. Veterinary Pathology, 35: 7476.

Jarrett, W. F. H., Crighton, G. W., and Dalton, R. G. 1966. Leukaemia and lymphosarcoma in animals and man. I. Lymphosarcoma or leukaemia in domestic animals. Veterinary Record, 79 (23): 693 .

Langenbach, A., McManus, P. M, Hendrick, M. J.,Shofer, F.S., and Sorenmo, K. U. 2001. Sensitivity and specificity of methods of assessing the regional lymph nodes for evidence of metastasis in dogs and cats with solid tumors. Journal of the American Veterinary Medical Association, 218(9): 1424-28.

Lumsden, J. H., and R, BAKER: 2000.Cytopathological techniques and interpretation In: Colour Atlas of the Cytology of the Dog and Cat. Mosby, London. pp. 7-20.

Marshak, R. R., Hare, W.C.D., Dutcher, R.M., Schwartzman, R.M., Switzer, J.W., and 
Hubben, K. 1966. Observations on a heifer with cutaneous lymphosarcoma. Cancer, 19(5): 724-734.

Marudhai, T., and Chidambaram, B. 2010. Cytological evaluation of canine lymphadenopathies-a review of 109 cases. VeterinarskiArhiv. 80(4): 499508.

Meinkoth, J. H., and R.L, Cowell.2002. Sample collection and preparation in cytology: Increasing diagnostic yield. Vet. Clin. North Am. Small Anim. Pract. 32: 11871207.

Morris, J. S., and J. M, Dobson.1992.Solid neoplasms. In Practice, 14: 18-24.

Murphy, M. C., Gomes, B. J., Kaczmarska, A., Hammond, G., and Sullivan, M. 2018. Evaluation of radiographs for the detection of sublumbar lymphadenopathy in dogs. In BSAVA Congress Proceedings 427-427.

Mylonakis, M.E., Borjesson, D.L., Leontides, L., Siarkou, V.I., Theodorou, K., and Koutinas, A.F. 2011.Cytologic patterns of lymphadenopathy in canine monocyticehrlichiosis. Veterinary clinical pathology, 40(1): 78-83.

Roszel, J F. 1981.Cytological procedures. JAAHA, 17: 903-910.

Salgado, B. S., Battaglia, C. T.,Stuchi, R. S., Cadioli, F. A., Rozza, D. B and Machado, G. F. 2011. What is your diagnosis? Lymphadenopathy in a cow with severe anemia. Veterinary clinical pathology, 40(1): 103.

Stahle, J.A., Larson, M.M., Rossmeisl, J.H., Dervisis, N., andNeelis, D. 2019. Diffusion weighted magnetic resonance imaging is a feasible method for characterizing regional lymph nodes in canine patients with head and neck disease. Veterinary Radiology \& Ultrasound, 60(2):176-183.

Strandstrom, H. V., and Bowen, J.M. 1982.
Canine leukemia-lymphoma complex: a model for human hematopoietic malignancies. In: Advances in Comparative Leukemic Research. Yohn DS, Blakerslee JR (eds). Elsevier New York: 447-50.

Teske, E. 1994. Canine malignant lymphoma: a review with comparison with human non-Hodgkin's lymphoma. Veterinary Quarterly, 16: 209-219.

Thangapandiyan, M., and Balachandran, C.2010. Cytological evaluation of canine lymphadenopathies-a review of 109 cases. Veterinarskiarhiv, 80(4): 499-508.

Thangapandiyan, M., and Balachandran, C. 2013. Infectious Agents Induced Lymphadenopathies in Dogs. Indian Veterinary Journal. 90(10): 80-81.

Thangapandiyan, M., and Balachandran, C. 2014. A comparative study on lymphadenopathy in dogs by cytology and histopathology.

Thangapandiyan, M., Balachandran, C., and MuraliManohar, B.2013. Incidence and haemato-biochemical changes in canine lymphoma. Tamilnadu Journal of Veterinary and Animal Sciences. 9(1): 29-31.

Villiers, E., and J, Dunn. 1988. Collection and preparation of smears for cytological examination. In Practice, 20: 370-377.

Wei, SH. E. M., Griffon and Whitbread, J.1999. Plexiform vascularisation of a retropharynigeal lymph node in a cat. Journal of Small Animal Practice. 40: 291-293.

Weller, R. E, C. A Holmberg., G. H,Thelen., and B.R, Madewell. 1982. Canine lymphosarcoma and hypercalcaemia: Clinical, laboratory and pathologic evaluation of twenty four cases. Journal of Small Animal Practice,23: 649-658. 


\section{How to cite this article:}

Avantika Sharma. 2020. Comparative Studies on Lymphadenopathies in Canines and Bovines. Int.J.Curr.Microbiol.App.Sci. 9(11): 2172-2180. doi: https://doi.org/10.20546/ijcmas.2020.911.258 